\title{
Isometric performance following total hip arthroplasty and rehabilitation
}

\author{
Karen L. Frost, PhD; ${ }^{1 *}$ Gina E. Bertocci, PhD; ${ }^{2}$ Craig A. Wassinger, MS, PT; ${ }^{3}$ Michael C. Munin, MD; ${ }^{3-4}$ \\ Ray G. Burdett, PhD, PT; ${ }^{3}$ Shirley G. Fitzgerald, $\mathbf{P h D}^{1,5}$ \\ ${ }_{1}^{1}$ Injury Risk Assessment and Prevention Laboratory and ${ }^{2}$ Department of Mechanical Engineering, University of Louis- \\ ville, Louisville, KY; ${ }^{3}$ School of Health and Rehabilitation and ${ }^{4}$ Department of Physical Medicine and Rehabilitation, \\ University of Pittsburgh, Pittsburgh, PA; ${ }^{5}$ Department of Veterans Affairs Pittsburgh Healthcare System, Pittsburgh, PA
}

\begin{abstract}
We compared differences in isometric strength between older adults who have undergone elective unilateral total hip arthroplasty (THA) and completed rehabilitation with a population of community-dwelling older adults who have not had THA. The study was a cross-sectional design, and 22 unilateral THA subjects and 38 community-dwelling older adults participated. THA subjects received on average 13 outpatient or home-based physical therapy sessions before evaluation. THA subjects were evaluated 4 to 5 months postsurgery. We assessed isometric muscle strength by measuring peak hip torque per body weight with a robotic dynamometer during abduction, flexion, and extension. No significant performance differences were observed between operated and nonoperated hips of THA subjects. THA subject operated and nonoperated hips generated significantly less peak torque per body weight during flexion $(p=0.03)$ compared with community-dwelling older adult hips (THA subject operated hips $=6.96 \mathrm{ft}-\mathrm{lb} / \mathrm{lb}$, THA subject nonoperated hips $=8.26 \mathrm{ft}-\mathrm{lb} / \mathrm{lb}$, communitydwelling older adult hips $=11.56 \mathrm{ft}-\mathrm{lb} / \mathrm{lb}$ ). . No significant differences were observed between THA subjects and community-dwelling older adults during hip extension $(p=0.55)$ or abduction $(p=0.17)$. At 4 to 5 months postsurgery, THA subjects were not at the same level of biomechanical performance as community-dwelling older adults. Significant strength deficits were found in THA subject operated versus nonoperated hips during isometric flexion. Additional or modified physical therapy that targets the hip flexors is recommended after THA.
\end{abstract}

Key words: arthroplasty, biomechanical performance, hip replacement, hip strength, isometric performance, muscle strength, osteoarthritis, physical therapy, rehabilitation, strength deficit.

\section{INTRODUCTION}

Osteoarthritis (OA) accounts for more dependency in walking, stair climbing, and other lower-extremity tasks than any other disease [1]. The past decade has seen an increase in the number of total hip arthroplasties (THAs) being performed for advanced cases of OA that involve severe pain and functional limitations. Recent reports indicate that in 1999, 168,000 THAs were performed in the United States [2]. As the number of aging adults in the United States continues to increase, we can reasonably anticipate a corresponding growth in the number of THAs based on current demographics [3] and past trends.

Current knowledge of patient outcomes following THA and rehabilitation does not provide consistent data for assessing THA outcomes. Studies based on selfreport data indicate that THA successfully alleviates pain and improves function relative to presurgical levels [4-6]. However, studies based on objective performance-based

\footnotetext{
Abbreviations: $\mathrm{CI}=$ confidence interval, $\mathrm{ICC}=$ intraclass correlation coefficient, $\mathrm{OA}=$ osteoarthritis, $\mathrm{ROM}=$ range of motion, THA = total hip arthroplasty.

* Address all correspondence to Karen L. Frost, PhD; University of Louisville, Injury Risk Assessment and Prevention Laboratory, 500 S. Preston Street, Room 204 Research Tower, Louisville, KY 40202; 502-852-0296; fax: 502-8520390. Email: k.frost@louisville.edu

DOI: 10.1682/JRRD.2005.06.0100
} 
measures indicate that despite postoperative improvements, THA subjects continue to exhibit deficits in strength [7-10], postural stability [2], and gait [11].

The ability to safely and independently walk, rise from a chair, or climb stairs contributes to independence and quality of life. Lower-extremity strength of the hip and knee is a significant predictor of the ability to perform these activities [1,12-13]. In a study examining the influence of hip and knee strength and hip power, Bean et al. found that isometric hip extensor strength was the strongest predictor of the amount of time that functionally impaired adults would require to complete five chair rises [12]. Isometric hip strength was also a significant predictor of timed stair climbing, balance, and habitual gait. Daubney and Culham examined lower-extremity muscle force and balance and found that isometric hip extensor strength was significantly lower in individuals who reported more falls [14]. Hip abductor weakness is also a major contributor to falls in older adults [15], and the importance of hip abductor strength in maintaining balance during gait is well established [16-18]. Increased hip flexion moment is associated with increased total hip joint force during stair climbing [19]. The demand placed on the hip muscles during activities such as stair climbing, walking, or rising from a chair could be problematic given Reardon et al.'s findings of significant quadriceps muscle wasting and decreased concentric peak torque generation 5 months after THA [8].

Previous studies have focused on comparing the performance of THA subject operated versus nonoperated hips as a means of evaluating surgical outcomes and prosthesis selection [9,20-21]. These studies report mixed results and, in several cases, lack statistical significance. Shih et al. reported a significant difference between operated and nonoperated hips in isometric flexion, extension, and abduction postoperatively at both 6 months and 1 year [7]. Long et al. found no significant difference between operated and nonoperated hip strength over a 2-year postoperative period [20], whereas Borja et al. found a significant difference in isokinetic abduction but not isometric adduction between operated and nonoperated hips [9].

We believe that differences between hip performance in THA subjects who have completed rehabilitation and community-dwelling older adults who have not undergone THA are more relevant for determining the effects of rehabilitation. Reardon et al. evaluated quadriceps strength of unilateral THA subjects using robotic dynamometry pre- and postoperatively at 5 months and compared these results with age-matched community-dwelling older adults $(n=12)$ [8]. Compared with preoperative conditions, THA subjects showed mild improvement in the nonoperated hip (concentric peak torque) yet no significant improvement in the operated hip, despite participation in physical therapy. Compared with communitydwelling older adults, patients in Reardon's study had significantly reduced quadriceps concentric peak torque $(p<0.008)$ but no difference in eccentric peak torque. Bertocci et al. evaluated isokinetic flexion, extension, and abduction performance in months 4 and 5 following surgery and compared THA subject performance with community-dwelling older adults [10]. THA subject operated versus nonoperated hips showed no significant differences in isokinetic performance for any of the examined variables; however, compared with community-dwelling older adults, THA subjects generated significantly less peak torque and work across all test conditions [10].

Because the nature of strength deficits following THA is still unresolved and relatively few studies have compared strength deficits of THA subjects with community-dwelling older adults, we conducted this study to further examine deficits following THA and rehabilitation. We sought to examine differences in isometric strength between THA subject operated and nonoperated hips and compare isometric strength between THA subjects and community-dwelling older adults as a means of providing comparative data for therapists treating THA subjects.

\section{METHODS}

\section{Subjects}

Sixty individuals gave written informed consent to participate in this study in accordance with University of Pittsburgh Institutional Review Board procedures. THA subjects were identified and recruited through physician offices or physical therapy departments within the University of Pittsburgh Medical Center. Community-dwelling older adults were recruited with paid advertisements placed in a local newspaper. All subjects were between 55 and 75 years. Inclusion criteria specified individuals who did not use an ambulatory assistive device, reported no history of major cardiovascular events in the previous 6 months, and were not taking corticosteroids. In addition, 
blood pressure and heart rate of subjects were within normal ranges. Potential participants were excluded for a positive history of neurological disorder, stroke, or transient ischemic attack. Community-dwelling older adults reported no hip arthritis or discrete hip pathology. THA subjects had a diagnosis of OA as the primary cause for elective THA surgery and were excluded for a positive history of surgical complication (dislocation, prostheses infection, procedure-associated neuropathy, or revision arthroplasty).

Each subject participated in one test session. THA subjects participated between 4 and 5 months postsurgery. As indicated previously [10], this time period was chosen for several reasons: (1) postoperative healing was significantly complete, (2) hip range of motion (ROM) precautions were typically relaxed by the orthopedic surgeon, (3) risk of dislocation was significantly reduced, (4) patients had resumed activities of daily living, and (5) patients were able to execute the tests included in the study protocol. We recruited 22 THA subjects and 38 communitydwelling older adults. The groups were similar in age, sex distribution, number of comorbidities, and domestic living status. The majority of subjects were Caucasian. The number of comorbidities for each group ranged from 1.4 to 2.0, excluding the presence of OA. Although surgical approach was not specified as an inclusion criterion, all THA subjects in this study had either a posterolateral or straight-lateral approach. THA subjects self-reported no discrete pathology of their contralateral hip.

Medical records revealed the average length of stay for surgery and acute care was 4 days for THA subjects. All but two THA subjects were discharged directly to an inpatient rehabilitation facility, where the average length of stay was 8 days. Following inpatient rehabilitation, THA subjects received an average of 13 sessions of outpatient or home-based physical therapy focused on increasing lower-extremity ROM and muscle strength and maximizing independence in performing mobility tasks and other activities of daily living. Therapeutic exercises were within the scope of standard outpatient physical therapy protocols for THA [22-23].

\section{Isometric Testing}

Isometric measurements were assessed with a Biodex System III Isokinetic Dynamometer version 3.03 (Biodex Medical Inc, Shirley, New York). Subjects were positioned on the dynamometer in a supine position for hip flexion and extension measurements and on their side for hip abduction measurement. Although hip precautions had been relaxed, we took a conservative approach and observed precautionary measures during testing; i.e., no hip flexion greater than $90^{\circ}$, no hip adduction past neutral, and no internal rotation past neutral. Subjects were properly positioned by a physical therapist before each test. To stabilize the body and minimize muscle compensation, we secured subjects to the platform with a 6 in.wide foam-padded strap positioned above the iliac crests. The left and right hips were assessed in communitydwelling older adults, and the operated and nonoperated hips were assessed in THA subjects. The greater trochanter was used as the bony landmark for matching the hip joint axis of rotation with the center of rotation on the dynamometer arm. We obtained gravitational correction values before each test by measuring the torque exerted on the dynamometer arm by the weight of the limb. The measured gravity effect was automatically accounted for in the output from the Biodex Advantage software program. Calibration of the Biodex dynamometer was performed according to the specifications outlined in the manufacturer's service manual [24].

Isometric strength data were collected over a $5 \mathrm{~s}$ maximal voluntary contraction during all tests. We assessed isometric peak torque per body weight for flexion, extension, and abduction to allow comparison between subjects. For hip flexion and extension, the hip was positioned at $45^{\circ}$ of flexion, and for abduction, the hip was positioned at $20^{\circ}$ of abduction. Selected hip placement positions were within the ROM for this age group and were in keeping with recommendations for isometric testing of the hip [25]. All subjects were able to accommodate this ROM without difficulty or adverse event. Subjects were provided with instructions prior to each test and encouraged to exert maximal effort during the test. Once instructed, subjects were permitted one practice trial. A waiting period of $1 \mathrm{~min}$ was observed between the practice trial and actual testing. No verbal encouragement was provided during testing.

\section{Data Analysis}

Parametric and nonparametric statistics were used for data analysis. We used paired $t$-tests to test for significant differences in isometric performance between THA subject operated and nonoperated hips because data were normally distributed and variances were homogenous. Isometric data of community-dwelling older adults were examined for bilateral differences between the right and 
left hip with the Wilcoxon signed rank test because data were not normally distributed and variances were nonhomogenous. Because no statistically significant difference was found, we calculated the average value of community-dwelling older adult right and left hips for subsequent use in between-group comparisons. We used nonparametric Mann-Whitney tests ( $U$ statistics) to compare THA subject and community-dwelling older adult hip performance because the groups were not equal in size and data were not normally distributed. An $\alpha$ level of 0.05 was set for all between-group comparisons. We estimated test-retest reliability of the dynamometer by calculating the intraclass correlation coefficient $\left(\mathrm{ICC}_{3,1}\right)$ for all hip biomechanical measures. Statistical analysis was performed with Statistical Package for the Social Sciences, version 11 (SPSS Inc., Chicago, Illinois).

\section{RESULTS}

\section{Reliability}

The ICC $_{3,1}$ for Biodex dynamometer measurements of peak torque per body weight during flexion, extension, and abduction ranged from 0.87 to 0.97 . In general, reliability coefficients above 0.75 indicate good reliability [26]. Therefore, the Biodex dynamometer has been justified as an acceptable measurement tool for evaluating hip musculature performance when used according to our study protocol.

\section{Demographics}

Table 1 shows the comparison of select demographic characteristics for both groups. No significant differences were noted between groups in age, sex, race/ethnicity, and mean number of comorbidities. No subjects reported pain prior to, during, or after testing procedures.

\section{Isometric Performance}

One THA subject was unable to complete an abduction test with his or her operated hip. On five occasions Biodex equipment or computer software problems were experienced. As a result, one or more isometric tests could not be completed (one of these occasions involved testing of a operated hip and four occasions involved testing of nonoperated hips), and data were not available for analysis. Resulting variations in sample sizes are indicated in the figures.

\section{THA Subject Operated vs Nonoperated Hips}

No significant difference was noted in isometric performance of THA subject operated versus nonoperated hips in either flexion ( $p=0.23)$, extension $(p=0.87)$, or abduction $(p=0.99)$. Mean and confidence interval (CI) values are shown in Table 2. THA subjects generated the highest values of peak torque per body weight during extension, followed by abduction and flexion.

\section{THA Subject Operated vs Community-Dwelling Older Adult Hips}

THA subject operated hips generated less peak torque per body weight during flexion, extension, and abduction compared with community-dwelling older adult hips. A significant difference was noted $(p=0.03)$ between flexion mean peak torque per body weight generated by THA subject operated hips (6.96 ft•lb/lb, CI: 4.89 to 9.03) and community-dwelling older adult hips $(11.56 \mathrm{ft} \bullet \mathrm{lb} / \mathrm{lb}$, CI: 9.17 to 13.96). Community-dwelling older adults generated 66 percent greater peak torque in flexion (Table 2). For extension, peak torque per body weight was 14.8 percent greater for community-dwelling older adult hips (53.22 ft•lb/lb, CI: 44.78 to 61.66) compared with THA subjects (46.35 ft•lb/lb, CI: 38.51 to 54.18 ), but this difference was not statistically significant $(p=0.54)$. For abduction, peak torque per body weight was 18 percent greater for community-dwelling older adult hips (18.36 ft•lb/lb, CI: 15.55 to 21.17) compared with THA subject, operated hips (15.52 ft•lb/lb, CI: 12.12 to 18.91), though again, this difference was not statistically significant $(p=0.17)$. Mean and CI values are shown in the Figure.

\section{THA Subject Nonoperated vs Community-Dwelling Older Adult Hips}

Comparison of THA subject nonoperated hips with community-dwelling older adult hips yielded results similar to the comparison of THA subject operated hips and community-dwelling older adult hips. THA subject nonoperated hips generated less peak torque per body weight during all test conditions compared with communitydwelling older adult hips. A significant difference was seen only during performance of isometric flexion. In flexion, mean peak torque per body weight was 40 percent greater for community-dwelling older adult hips $(11.56 \mathrm{ft} \bullet \mathrm{lb} / \mathrm{lb}$, CI: 9.17 to 13.96$)$ compared with THA subject nonoperated hips (8.26 ft•lb/lb, CI: 5.58 to 10.94), and this difference was significant $(p=0.03)$. For extension, peak torque per body weight was 17 percent greater for 
Table 1.

Demographic characteristics for total hip arthroplasty (THA) subjects $(n=22)$ and community-dwelling older adults $(n=38)$.

\begin{tabular}{lcc}
\hline \multicolumn{1}{c}{ Characteristic } & THA Subjects & Community-Dwelling Older Adults \\
\hline Age (yr) (mean \pm SD) & $68 \pm 5$ & $65 \pm 6$ \\
Age Range (yr) & 56 to 75 & 55 to 75 \\
Osteoarthritis (yr w/ diagnosis) (mean \pm SD) & $8 \pm 7$ & - \\
Comorbidities* (No.) & 2 & 5.4 \\
Female (\%) & 60 & 55 \\
Race/Ethnicity (\% Caucasian) & 100 & 92 \\
Domestic Status (\%) & & 63 \\
Married/Partner & 86 & 29 \\
Divorced/Widowed & 14 & 8 \\
Never Married & 0 & \\
\hline
\end{tabular}

Note: No significant differences in demographics between groups were present.

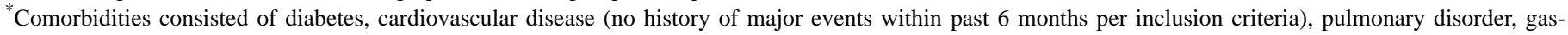
trointestinal disorder, cancer, thyroid disorder, renal disorder, neurological disorder, psychiatric disorder, or other specified by subject.

$\mathrm{SD}=$ standard deviation .

Table 2.

Mean peak torque per body weight $(\mathrm{ft} \cdot \mathrm{lb} / \mathrm{lb})$ and 95 percent confidence interval $(\mathrm{CI})$ for total hip arthroplasty subject operated and nonoperated hips vs community-dwelling older adult hips for flexion, extension, and abduction.

\begin{tabular}{|c|c|c|c|}
\hline Variable & Hip Joint & $\begin{array}{l}\text { Peak Torque/Body Weight } \\
\text { (ft•lb/lb) }\end{array}$ & $95 \%$ CI \\
\hline \multirow{3}{*}{ Flexion } & Operated & $6.96^{*}$ & 4.89 to 9.03 \\
\hline & Nonoperated & 8.26 & 5.58 to 10.94 \\
\hline & Community-dwelling older adult & 11.56 & 9.17 to 13.96 \\
\hline \multirow[t]{2}{*}{ Extension } & Operated & 46.35 & 38.51 to 54.18 \\
\hline & Nonoperated & 45.42 & 34.63 to 56.21 \\
\hline \multirow[t]{3}{*}{ Abduction } & Operated & 15.52 & 12.12 to 18.91 \\
\hline & Nonoperated & 12.98 & 8.85 to 17.10 \\
\hline & Community-dwelling older adult & 18.36 & 15.55 to 21.17 \\
\hline
\end{tabular}

community-dwelling older adult hips $(53.22 \mathrm{ft} \bullet \mathrm{lb} / \mathrm{lb}$, CI: 44.78 to 61.66$)$ versus THA subject nonoperated hips (45.42 ft•lb/lb, CI: 34.63 to 56.21), and for abduction, peak torque per body weight was 41 percent greater for community-dwelling older adult hips $(18.36 \mathrm{ft} \bullet \mathrm{lb} / \mathrm{lb}, \mathrm{CI}$ : 15.55 to 21.17) compared with THA subject nonoperated hips $(12.98 \mathrm{ft} \cdot \mathrm{lb} / \mathrm{lb}, \mathrm{CI}: 8.85$ to 17.10$)$. The results for isometric extension and abduction were not statistically significant ( $p=0.55$ and $p=0.17$, respectively). Mean and CI values are shown in the Figure.

\section{DISCUSSION}

Few studies have investigated hip strength postrehabilitation for the purpose of comparing THA and reha- bilitation outcomes with similarly aged communitydwelling adults. These findings are important because they indicate a substantial deficit in isometric strength in THA subjects compared with community-dwelling older adults at a time shortly after the subjects had completed rehabilitation. Despite patient self-reports of decreased pain and improved function relative to presurgical levels [27-28], our current and previous findings [10] and those of Reardon et al. [8] and others [29] suggest that compared with community-dwelling older adults, THA subjects continue to experience strength deficits. THA subjects' hip strength was only 60 percent of that generated by community-dwelling older adults in flexion and 87 percent and 84 percent of the strength generated by community-dwelling older adults in extension and abduction, respectively. In flexion, this difference was 
JRRD, Volume 43, Number 4, 2006

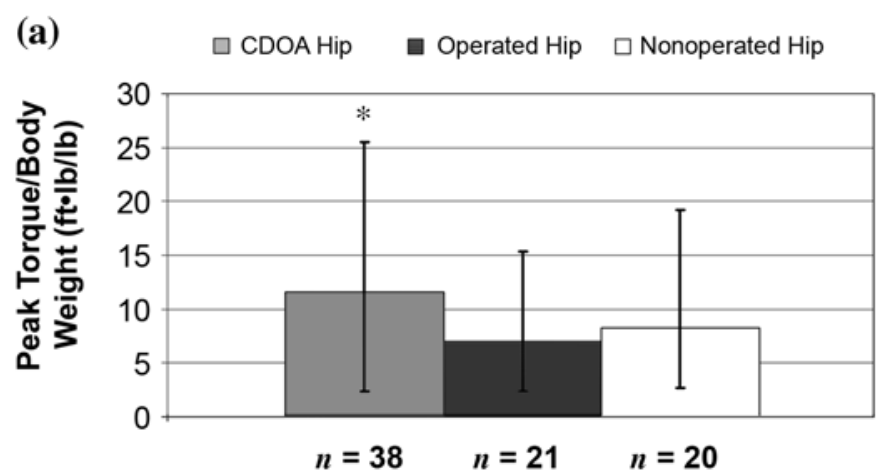

(b)
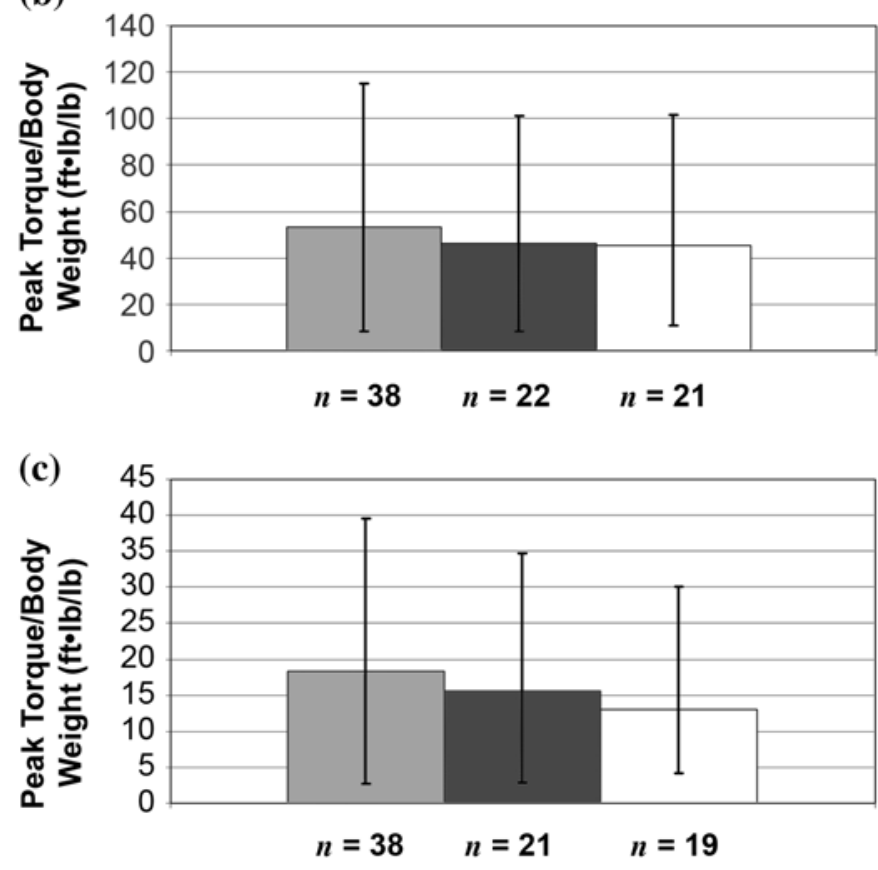

Figure.

Mean peak torque per body weight $(\mathrm{ft} \bullet \mathrm{lb} / \mathrm{lb})$ for isometric (a) flexion, (b) extension, and (c) abduction for community-dwelling older adults (CDOAs) and both operated and nonoperated hips of total hip arthroplasty (THA) subjects. Error bars indicate 95\% confidence interval for each data set. *Significant differences were noted between hip performance of CDOAs and both operated and nonoperated hip performance of subjects with THA $(p=0.03)$.

significant, which indicates that at 4 to 5 months postsurgery, hip strength in THA subjects has not been restored to levels comparable with community-dwelling older adults.

This study looked at subjects 4 to 5 months following THA surgery. At this time, surgical ROM restrictions are likely relaxed and postoperative rehabilitation complete. Until this time, hip adduction and internal rotation past neutral is generally restricted, as is hip flexion motion greater than $90^{\circ}$. If strength training had occurred for hip flexion, it would not have been conducted through a full ROM, which may have contributed to the decreases in flexor strength seen in this study. During rehabilitation, hip flexion is not as challenged as hip extension and abduction. Additionally, prescribed use of a cane postoperatively decreases both abductor and flexion moments about the operated hip [30]. This reduction in hip joint moments during postoperative gait may promote flexor and abductor muscle atrophy and associated decreases in strength. Thus, hip flexion may not be specifically targeted during postoperative strength or gait training, and patients may undergo disuse atrophy because of cane use and/or hip precautions. We should also note that strength testing was not performed before surgery. Therefore, we do not know if differences in hip flexion between groups were present prior to surgery and were caused by the OA that resulted in the THA.

Weakness of the hip flexors may contribute to difficulty during normal activities of daily living. Luepongsak et al. reported substantial hip flexion moments during walking, chair rising, stair descent, and trunk bending [31]. Shih et al. indicated that compared with the extensors and abductors, the flexors have the slowest recovery in the first year following THA [7]. Our findings and those of Shih et al. suggest that current rehabilitation protocols may not adequately restore hip flexor strength.

Isometric hip strength plays an important role in gait by contributing to trunk control and stability [16-17]. In a study of 15 THA subjects who were an average of 23 months post-THA, Sicard-Rosenbaum et al. reported isometric muscle weakness of the quadriceps, abductors, and extensors as well as significantly decreased gait speed in THA subjects compared with age- and sex-matched control subjects [29]. Hip patients in Sicard-Rosenbaum et al.'s study not only exhibited significant decreases in gait speed compared with control subjects but also were unable to walk fast enough to cross an intersection safely [29]. Weak isometric hip abductor and extensor strength has been implicated as a major contributor of increased falls [14], inability to safely rise from a chair, and difficulty ascending and descending stairs [12]. Normal stair climbing results in large hip flexion moments [19] and generates large hip loads.

We also found no significant difference in isometric performance between THA subject operated and nonoperated hips. This finding is consistent with isometric performance studies conducted by Borja et al. [9] and 
Trudelle-Jackson et al. [2] and with Long et al.'s [20] findings from strength data derived from force plate measurements. The data from these studies suggest that after THA, hip strength is equally poor in both hips at time periods ranging from 4 to 12 months postsurgery. We believe that this symmetrically poor performance may be a result of reduced mobility and functional limitations imposed by the operated hip, which lead to disuse atrophy and muscle weakness in the nonoperated hip before and after surgery. However, our findings differ from Shih et al.'s finding of significantly decreased isometric flexion in the operated versus nonoperated hip at 6 months and 1 year following THA [7]. This difference may be attributable to a combination of factors that differ between subjects in our study and those in Shih et al.'s study. For example, Shih et al.'s patients did not receive rehabilitation after THA, the subject age range was 24 to 71 , and male subjects in his study received THA because of osteonecrosis associated with alcoholism.

Bertocci et al. previously reported significant deficits at 4 to 5 months postsurgery during isokinetic flexion, extension, and abduction in THA subjects compared with community-dwelling older adults [32]. Bertocci et al.'s study was based on a subset of our study's sample population. These two studies indicate that after completion of rehabilitation, THA subjects continue to exhibit deficits in both muscle strength and dynamic neuromuscular function. Isometric deficits appear to be greatest for the hip flexors, whereas isokinetic deficits are significant across all muscle groups. These findings, in part, support the rationale of Trudelle-Jackson et al. in providing a late-phase exercise intervention initiated 4 to 12 months after THA that emphasizes controlled weight-bearing and is aimed at improving postural stability. Weak dynamic strength in the hip abductors and extensors and, to a lesser extent, the hip flexors, contributes to postural instability in THA subjects [2]. Trudelle-Jackson et al. found that patients who received the late-phase exercise program showed significant improvement in postural stability and hip strength compared with THA subjects who participated in a program of basic isometric and active ROM exercises similar to those provided during the early stage of a typical rehabilitation protocol for THA.

A limitation of this study is that we did not evaluate functional status to determine if a direct link existed between isometric strength and functional tasks and activities of daily living performance. Although the evidence indicates that deficits exist in the short term fol- lowing rehabilitation, a rigorous assessment of the effect of current rehabilitation protocols on physical functioning is needed to guide clinicians in developing more effective protocols that improve recovery and maximize long-term postoperative functioning. Another limitation of this study was that tests were not performed in random order. The same order of progression (flexion, extension, abduction) was used for all subjects, and fatigue may have had an effect on the relative ranking of strength across the various muscle groups. However, a significant difference in flexion was found despite it being the exercise performed first. In addition, each THA subject was tested on the operated hip first. This order could present an advantage to the nonoperated hip because of a learning affect. However, our findings did not reveal an advantage given that nonoperated hip performance was statistically equivalent to operated hip performance. Another factor that was not controlled for in this study was preoperative strength. This factor should be considered in future studies. Additionally, future studies could be strengthened by randomizing the order of testing and using age- and sex-matched control subjects.

\section{CONCLUSIONS}

OA of the hip results in reduced levels of patient activity over a prolonged period of time. Following surgical intervention and rehabilitation, patients report decreased pain and improved function relative to presurgical levels; however, isometric strength deficits persist and are significant in flexion compared with communitydwelling older adults. These deficits indicate that rehabilitation protocols are not restoring THA subjects to a level similar to community-dwelling older adults. These results can be used by clinicians and therapists to improve current rehabilitation protocols.

\section{ACKNOWLEDGMENTS}

We would like to acknowledge the following individuals and groups for their assistance with subject recruitment: Raj K. Sinha, MD; Lawrence Crossett, MD; and staff at the Division of Adult Reconstructive Surgery, University of Pittsburgh; Brian Urso, MS, OTR/L, and therapists at the University of Pittsburgh Medical Center Rehabilitation Hospital; and Edward McClain III, MD, 
and staff at Three Rivers Orthopedic Associates. We thank the Department of Veterans Affairs Pittsburgh Healthcare System Human Engineering Research Laboratory for the use of its laboratory and Biodex equipment. Fernando Aguel, MSBE, and Dongran $\mathrm{Ha}, \mathrm{PhD}$; are acknowledged for their assistance with testing.

This material is the result of work supported with resources of the Arthritis Foundation, the Western Pennsylvania Arthritis Foundation, and the University of Pittsburgh Arthritis Institute. Opinions expressed are those of the authors and do not necessarily represent those of the funding agencies.

The authors have declared that no competing interests exist.

\section{REFERENCES}

1. Guccione AA, Felson DT, Anderson JJ, Anthony JM, Zhang Y, Wilson PW, Kelly-Hayes M, Wolf PA, Kreger BE, Kannel WB. The effects of specific medical conditions on functional limitations of elders in the Framingham Study. Am J Public Health. 1994;84(3):351-58. [PMID: 8129049]

2. Trudelle-Jackson E, Emerson R, Smith S. Outcomes of total hip arthroplasty: A study of patients one year postsurgery. J Orthop Sports Phys Ther. 2002;32(6):260-67. [PMID: 12061707]

3. Weaver FM, Hughes SL, Almagor O, Wixson R, Manheim L, Fulton B, Singer R. Comparison of two home care protocols for total joint replacement. J Am Geriatr Soc. 2003; 51(4):523-28. [PMID: 12657073]

4. Shields RK, Enloe LJ, Leo KC. Health related quality of life in patients with total hip or knee replacement. Arch Phys Med Rehabil. 1999;80(5):572-79. [PMID: 10326924]

5. Nilsdotter AK, Roos EM, Westerlund JP, Roos HP, Lohmander LS. Comparative responsiveness of measures of pain and function after total hip replacement. Arthritis Rheum. 2001;45(3):258-62. [PMID: 11409667]

6. Munin MC, Kwoh CK, Glynn NW, Crossett LS, Rubash HE. Early inpatient rehabilitation after elective hip and knee arthroplasty. JAMA. 1998;279(11):847-52. [PMID: 9515999]

7. Shih $\mathrm{CH}, \mathrm{Du} \mathrm{YK}$, Lin YH, Wu CC. Muscular recovery around the hip joint after total hip arthroplasty. Clin Orthop Relat Res. 1994;(302):115-20. [PMID: 8168288$]$

8. Reardon K, Galea M, Dennett X, Choong P, Byrne E. Quadriceps muscle wasting persists 5 months after total hip arthroplasty for osteoarthritis of the hip: A pilot study. Intern Med J. 2001;31(1):7-14. [PMID: 11479364]
9. Borja F, Latta LL, Stinchfield FE, Obreron L. Abductor muscle performance in total hip arthroplasty with and without trochanteric osteotomy. Clin Orthop Relat Res. 1985; (197):181-90. [PMID: 4017333]

10. Bertocci GE, Munin MC, Frost KL, Burdett RG, Wassinger CA, Fitzgerald SG. Isokinetic performance after total hip replacement. Am J Phys Med Rehabil. 2004; 83(1):1-9. [PMID: 14709968]

11. Perron M, Malouin F, Moffet H, McFadyen BJ. Threedimensional gait analysis in women with a total hip arthroplasty. Clin Biomech (Bristol, Avon). 2000;15(7):504-15. [PMID: 10831810]

12. Bean JF, Leveille SG, Kiely DK, Bandinelli S, Guralnik JM, Ferrucci L. A comparison of leg power and leg strength within the InCHIANTI study: Which influences mobility more? J Gerontology A Biol Sci Med Sci. 2003; 58(8):728-33. [PMID: 12902531]

13. Schenkman M, Hughes MA, Samsa G, Studenski S. The relative importance of strength and balance in chair rise by functionally impaired older individuals. J Am Geriatr Soc. 1996;44(12):1441-46. [PMID: 8951313]

14. Daubney ME, Culham EG. Lower-extremity muscle force and balance performance in adults aged 65 years and older. Phys Ther. 1999;79(12):1177-84. [PMID: 10630286]

15. Shumway-Cook A, Gruber W, Baldwin M, Liao S. The effect of multidimensional exercises on balance, mobility, and fall risk in community-dwelling older adults. Phys Ther. 1997;77(1):46-57. [PMID: 8996463]

16. Vaz MD, Kramer JF, Rorabeck CH, Bourne RB. Isometric hip abductor strength following total hip replacement and its relationship to functional assessments. J Orthop Sports Phys Ther. 1993;18(4):526-31. [PMID: 8220410]

17. Loizeau J, Allard P, Duhaime M, Landjerit B. Bilateral gait patterns in subjects fitted with a total hip prosthesis. Arch Phys Med Rehabil. 1995;76(6):552-57. [PMID: 7763155]

18. Mochon S, McMahon TA. Ballistic walking. J Biomech. 1980;13(1):49-57. [PMID: 7354094]

19. Shelley FJ, Anderson DD, Kolar MJ, Miller MC, Rubash HE. Physical modeling of hip joint forces in stair climbing. Proc Inst Mech Eng [H]. 1996;210(1):65-68. [PMID: 8663894]

20. Long WT, Dorr LD, Healy B, Perry J. Functional recovery of noncemented total hip arthroplasty. Clin Orthop Relat Res. 1993;(288):73-77. [PMID: 8458156]

21. Mostardi RA, Askew MJ, Gradisar IA Jr, Hoyt WA Jr, Snyder $\mathrm{R}$, Bailey B. Comparison of functional outcome of total hip arthroplasties involving four surgical approaches. J Arthroplasty. 1988;3(3):279-84. [PMID: 3183682]

22. Dutton M. Orthopaedic examination, evaluation, and intervention. New York (NY): McGraw-Hill Companies, Inc; 2004. p. 1459. 
23. Burton DS, Imrie SH. Total hip arthroplasty and postoperative rehabilitation. Phys Ther. 1973;53(2):132-40. [PMID: 4685033]

24. Biodex Medical Systems Inc. Biodex System 3 Pro Manual. Brookhaven (NY): Biodex Medical Systems Inc; 1999.

25. Callahan LF. Impact of rheumatic disease on society. In: Wegner ST, Belza BL, Gall EP, editors. Clinical care in the rheumatic diseases. Atlanta (GA): American College of Rheumatology; 1996. p. 209-13.

26. Portney LG, Watkins MP. Foundations of clinical research: Applications to practice. 2nd ed. New Jersey: Prentice Hall Health; 2000. p. 565.

27. Fitzgerald JD, Orav EJ, Lee TH, Marcantonio ER, Poss R, Goldman L, Mangione CM. Patient quality of life during the 12 months following joint replacement surgery. Arthritis Rheum. 2004;51(1):100-109. [PMID: 14872462]

28. Laupacis A, Bourne R, Rorabeck C, Feeny D, Wong C, Tugwell P, Leslie K, Bullas R. The effect of elective total hip replacement on health-related quality of life. J Bone Joint Surg Am. 1993;75(11):1619-26. [PMID: 8245054]

29. Sicard-Rosenbaum L, Light KE, Behrman AL. Gait, lower extremity strength, and self-assessed mobility after hip arthroplasty. J Gerontol A Biol Sci Med Sci. 2002;57(1): M47-51. [PMID: 11773212]

30. Ajemian S, Thon D, Clare P, Kaul L, Zernicke RF, LoitzRamage B. Cane-assisted gait biomechanics and electromyography after total hip arthroplasty. Arch Phys Med Rehabil. 2004;85(12):1966-71. [PMID: 15605334$]$

31. Luepongsak N, Amin S, Krebs DE, McGibbon CA, Feson D. The contribution of type of daily activity to loading across the hip and knee joints in the elderly. Osteoarthritis Cartilage. 2002;10(5):353-59. [PMID: 12027536]

32. Bertocci GE, Munin MC, Frost KL, Burdett RG, Breisinger T. Assessment of muscle fatigue in total hip replacement (THR) patients as a means to evaluate rehabilitation status. In: Proceedings of the Physical Medicine and Rehabilitation Conference; 2001 Sep; New Orleans, LA. Chicago (IL): American Academy of Physical Medicine and Rehabilitation; 2001.

Submitted for publication June 15, 2005. Accepted in revised form December 30, 2005. 